\title{
Classical and Quantized Affine Models of Structured Media
}

\author{
Jan J. Sławianowski \\ Institute of Fundamental Technological Research, \\ Polish Academy of Sciences, \\ Świętokrzyska 21, 00-049 Warsaw, Poland \\ e-mail: jslawian@ippt.gov.pl
}

November 1, 2018

\begin{abstract}
Having in view some applications in nanophysics, in particular in nanophysics of materials, we develop new dynamical models of structured bodies with affine internal degrees of freedom. In particular, we construct some models where not only kinematics but also dynamics of systems of affine bodies is affinely invariant. Quantization schemes are developed. This is necessary in the range of physical phenomena we are interested in.
\end{abstract}

\section{Introduction}

The idea of microstructure is a rather old one and goes back to brothers Cosserat who formulated the theory of continuum consisting of infinitesimal gyroscopes 1. Eringen modified such a model by introducing homogeneous deformations as additional microstructural modes [2]. These theories were rather phenomenological and often motivated by some kind of mathematical (more precisely, differential-geometric) aesthetics. There was also mechanical motivation based on theory of granular media and continuous limit of the dynamics of molecular crystals.

Recently the interest in mechanics of structured media becomes more and more intensive in connection with nano-structures, various supramolecular structures, and defect theory. There are also some special problems like the dynamics of suspensions, gas bubbles in fluids, and some very peculiar models like kinetic media [3, 4, 5, 6, 7. In all these problems, in defect theory and in dynamics of fullerens, affine model of structural elements is well-motivated both from the physical and geometrical point of view. There are some interesting problems, completely new ones in comparison with traditional phenomenological mechanics of structured continua. First of all, in dynamics of strongly 
interacting systems of objects in nano-scale, on the classical level, the traditional scheme of constrained motion based on the d'Alembert principle does not seem reliable any longer. Instead, one should base the dynamics on some effective collective models motivated by appropriate symmetry demands. We believe, there are physical reasons to expect that the dynamics of internal and collective affine modes should be also affinely invariant. In traditional theories derived from the d'Alembert principle, the dynamics of affine modes is invariant only under the Euclidean group. Below we suggest some dynamical models of a system of bodies with affine degrees of freedom, with Hamiltonians invariant under the affine group. One can smoothly include also some terms invariant only under the Euclidean group, there is however a natural temptation to consider them as a merely perturbation to the background affine dynamics. Some arguments from the solid state physics, like the concept of effective mass support the idea that in complicated problems of condensed matter theory the "true" metric tensor is not necessarily a fundamental geometric background of equations of motion, Lagrangians and Hamiltonians (having in mind microphysical models, we assume Hamiltonian, variational dynamical models, without dissipation on the fundamental level).

There is also another important novelty in comparison with traditional macroscopic models. Namely, in the nano-scale the quantum background of the dynamics must be seriously taken into account. Because of this we developed some quantization scheme. But at the same time one uses the concepts of macroscopic origin. like, e.g., deformation tensors, deformation invariants, etc. It seems rather amazing that one formulates questions like: what is a complex quantum probability amplitude for the deformation tensors and deformation invariants to be found in some fixed range? What are the corresponding quantum transition probabilities? There are even more serious problems. Namely, in this very peculiar range of phenomena one has to do with a very complicated convolution of classical and quantum problems. In this, rather unexpected way, the old problems from the realm of foundations of quanta $[\underline{8}$, like decoherence, wave functions reduction, possibility of nonlinear quantum description, etc. revive as ones motivated by quite practical, structural physics. One must honestly say, there is more secrets than well-established facts and answers here.

\section{Affine bodies and their systems. General con- cepts and basic quantities}

There is no place here for the very detailed geometric description. A rather exhaustive treatment of the differential-geometric background of our ideas was presented in some earlier papers (cf. [9, 10, and references there) and a more detailed treatment will soon appear. Here we base mainly on the analytic description where the physical and material spaces are simply identified with $\mathbb{R}^{n}$ by some choice of orthonormal Cartesian coordinates. As far as possible we work in a non-specified dimension $n$ and only at some final stages we specify $n$ to its 
physical value 3 or to 2 and 1 , which obviously are also physically interpretable. This "false" generality is mathematically convenient and better reveals some structural features of the model, hidden behind the particular value $n=3$. Moreover, it suggests even some analytical solving procedures.

So, the body we consider consists of elements performing the translational motion in the physical space $\mathbb{R}^{n}$ (physically $n=3,2,1$ ) and also some internal motion. The latter is simply a relative motion of microconstituents; the translational $\mathbb{R}^{n}$-degrees of freedom are attributed to the centres of mass. But of course one can also admit situations when additional degrees of freedom are essentially internal ones, like, e.g., in spin media. And in any case the distinction between internal and relative motion may be (although need not be) historical, based on some conventions or on our laboratory abilities. From now on we do not discuss this problem and for brevity all non-translational degrees of freedom will be referred to as internal ones.

In mechanics of structureless continua the configuration space may be identified with $\operatorname{Diff}(n, \mathbb{R})$, i.e., the group of (sufficiently smooth) diffeomorphisms of $\mathbb{R}^{n}$ onto itself. Let us remember, according to our analytical conventions both the physical and material space are simply identified with $\mathbb{R}^{n}$. Any diffeomor$\operatorname{phism} \varphi \in \operatorname{Diff}(n, \mathbb{R})$ establishes an interrelation between Lagrangian (material) and Eulerian (physical, current) coordinates, respectively $a^{k}$ and $x^{i}$,

$$
x^{i}=\varphi^{i}\left(\ldots, a^{k}, \ldots\right) .
$$

In this way the configuration space of continuous medium is identified with the infinite-dimensional group $\operatorname{Diff}(n, \mathbb{R})$. In the Arnold description of incompressible ideal fluid [11] this group is constrained to $\operatorname{SDiff}(n, \mathbb{R})$, i.e., the subgroup of volume-preserving diffeomorphisms. Euler equations are interpreted in terms of right-invariant geodetic Hamiltonian systems on this group. This approach turned out to be at least heuristically effective in hydrodynamical problems.

Of course, $\operatorname{Diff}(n, \mathbb{R})$ would be completely non-effective and just meaningless as the configuration space of internal degrees of freedom of structured bodies. Nevertheless, there exist both physical and analytical reasons to concentrate on models with degrees of freedom based on appropriately chosen groups and their homogeneous spaces. From the physical and computational point of view it is clear that one must use some geometrically well-motivated finite-dimensional subgroups $G \subset \operatorname{Diff}(n, \mathbb{R})$, just Lie groups acting in $\mathbb{R}^{n}$. The most traditional pattern is $G=\mathrm{SO}(n, \mathbb{R})$, i.e., rigid body model of internal degrees of freedom. It is not excluded, especially in quantized theory that the non-connected configuration space $G=\mathrm{O}(n, \mathbb{R})$ with mirror-reflected configurations may be also acceptable. Other natural model with more degrees of freedom is that of affinelyrigid, i.e., homogeneously deformable, body; $G=\mathrm{GL}^{+}(n, \mathbb{R})$ or perhaps, as above, the total non-connected linear group $G=\mathrm{GL}(n, \mathbb{R})$, with reflections admitted. One can also think about $\mathrm{L}(n, \mathbb{R})$, the algebra of all $n \times n$ matrices as an admissible configuration space. Incidentally, there is a subtle difference between admitting mirror-reflected configurations of metrically- and affinely-rigid bodies. Namely, $\mathrm{GL}^{+}(n, \mathbb{R})$ and its mirror-reflected coset (not a subgroup!) $\mathrm{GL}^{-}(n, \mathbb{R})$ 
in $\mathrm{GL}(n, \mathbb{R})$ infinitesimally approach each other in $\mathrm{L}(n, \mathbb{R})$, being separated only by the $\left(n^{2}-1\right)$-dimensional subset of singular matrices in $\mathrm{L}(n, \mathbb{R})$. On the other hand, $\mathrm{SO}(n, \mathbb{R})$ and the set of improper rotations $\mathrm{O}(n, \mathbb{R}) \backslash \mathrm{SO}(n, \mathbb{R})$ (the complement of $\mathrm{SO}(n, \mathbb{R})$ in $\mathrm{O}(n, \mathbb{R}))$ are so-to-speak finitely separated in $\mathrm{L}(n, \mathbb{R})$.

There are also natural models placed between gyroscopic and affine degrees of freedom; one deals then with a constrained affinely-rigid body. Let us mention incompressible (isochoric) body when $G=\mathrm{SL}(n, \mathbb{R})$, the special linear group. Just as previously, one can also think about admitting mirror reflections. Then $G=\mathrm{UL}(n, \mathbb{R})=\{\varphi \in \operatorname{GL}(n, \mathbb{R}):|\operatorname{det} \varphi|=1\}$, the unimodular group. It is a union of $\operatorname{SL}(n, \mathbb{R})$ and of its coset in $\operatorname{GL}(n, \mathbb{R})$ consisting of matrices with the minus-one-determinant. Obviously, just as in the case of metrically-rigid body, the manifolds of proper and improper (orientation-changing) isochoric mappings are finitely-separated in $\mathrm{L}(n, \mathbb{R})$. In a sense an opposite model (less realistic) is that of shape-preserving affinely-rigid body when, roughly speaking, internal configurations are built of dilatations and rotations, $G=\mathbb{R}^{+} \mathrm{SO}(n, \mathbb{R}) ; \mathbb{R}^{+}$ denoting the multiplicative group of positive real numbers. Again, admitting orientation-preserving mappings we have $G=\mathbb{R}^{+} \mathrm{O}(n, \mathbb{R})$. And here also the two connected components approach each other infinitely close. The closure of $G$ contains the null matrix, therefore, it is not a subset of $\mathrm{GL}(n, \mathbb{R})$.

All these subgroups of $\operatorname{Diff}(n, \mathbb{R})$ are contained in $\operatorname{GL}(n, \mathbb{R})$; they consist of linear mappings. These mappings describe configurations in such a way that the material point with Lagrangian coordinates $a^{k}$ occupies the spatial position with Euler coordinates $y^{i}$,

$$
y^{i}=x^{i}+\varphi_{k}^{i} a^{k}
$$

where $x^{i}$ are spatial coordinates of the centre of mass. The very geometry suggests also some groups of non-affine transformations, e.g., the projective group $\operatorname{GPr}(n, \mathbb{R})$, conformal group $\operatorname{Co}(n, \mathbb{R})$. One can realize physical situations where the relevant collective modes of internal motion are described just by these groups. It is also non-excluded that the complex group $\mathrm{GL}(n, \mathbb{C})$ or its unitary subgroup $\mathrm{U}(n) \subset \mathrm{GL}(n, \mathbb{C})[12$ may be useful. In any case, it happens quite often in physics that the complexification leads to quite amazing, unexpected new results.

Just as in our earlier papers we concentrate here on the affine model, when $G=\mathrm{GL}(n, \mathbb{R})$. Incidentally, the projective model may be in some sense reduced to it, because $\operatorname{GPr}(n, \mathbb{R})$ is isomorphic with $\mathrm{SL}(n+1, \mathbb{R})$. In any case, affine modes seem to be dominant for small structure entities like molecules, microdefects, some supramolecular clusters, fullerens, etc.

The configuration space of a single structural elements is identified with

$$
Q=\mathbb{R}^{n} \times G=Q_{\mathrm{tr}} \times Q_{\mathrm{int}},
$$

in particular, for elements with affine modes of deformation:

$$
Q=\mathbb{R}^{n} \times \mathrm{GL}(n, \mathbb{R}) .
$$

Usually, especially in classical (non-quantized) problems, GL $(n, \mathbb{R})$ is replaced by $\mathrm{GL}^{+}(n, \mathbb{R})$. The labels "tr" and "int" refer obviously to translational and 
internal degrees of freedom. The total body (medium) consists of $N$ elements. Its configuration space is obviously given by the Cartesian product

$$
Q^{N}=Q_{\mathrm{tr}}^{N} \times Q_{\mathrm{int}}^{N} \simeq \mathbb{R}^{n N} \times G^{N} ;
$$

in our treatment $\mathrm{GL}(n, \mathbb{R})$ or $\mathrm{GL}^{+}(n, \mathbb{R})$ substituted for $G$. Therefore, the configuration is an array:

$$
q=\left(x_{1}, \ldots, x_{N} ; \varphi_{1}, \ldots, \varphi_{N}\right),
$$

where $x_{A} \in \mathbb{R}^{n}, \varphi_{A} \in \mathrm{GL}(n, \mathbb{R})$, and $A=\overline{1, N}$.

For a single structure element the summation of usual kinetic energies of its constituents gives in virtue of (2), the usual d'Alembert form

$$
T^{\mathrm{d}^{\prime} \mathrm{A}}=T_{\mathrm{tr}}^{\mathrm{d}^{\prime} \mathrm{A}}+T_{\mathrm{int}}^{\mathrm{d}^{\prime} \mathrm{A}}=\frac{M}{2} \operatorname{Tr}\left(v v^{T}\right)+\frac{1}{2} \operatorname{Tr}\left(\xi J \xi^{T}\right),
$$

where $v \in \mathbb{R}^{n}, \xi \in \mathrm{L}(n, \mathbb{R})$ denote respectively the translational and internal velocities:

$$
v^{i}=\frac{d x^{i}}{d t}, \quad \xi_{k}^{i}=\frac{d \varphi_{k}^{i}}{d t},
$$

and $M, J$ are constant inertial characteristics. More precisely, $M$ is the total mass of the element and the symmetric positively definite matrix $J$ is its modified inertial tensor, i.e., second-order moment of the mass distribution with respect to co-moving (Lagrange) coordinates,

$$
M=\sum_{p} \mu_{p}, \quad J^{k l}=\sum_{p} \mu_{p} a_{p}^{k} a_{p}^{l} .
$$

Summation is performed over constituents ("atoms") of the element ("molecule"); $\mu_{p}$ is the mass of the $p$-th constituent. Sometimes it is convenient to use the symbol of integration with respect to the mass distribution measure $\mu$ :

$$
J^{k l}=\int a^{k} a^{l} d \mu(a) .
$$

Remark: the kinetic energy (6) is spatially isotropic, i.e., invariant under the transformations $L_{A}$ below (14) with $A$ restricted to the orthogonal group $\mathrm{O}(n, \mathbb{R})$ (spatial rotations). So are its both terms separately. The material rotations $R_{A}$ in (14) preserve $T_{\mathrm{tr}}$ trivially, but in general $T_{\mathrm{int}}$ is non-invariant under the right-acting $\mathrm{O}(n, \mathbb{R})$. However, it is invariant under the right actions of $\mathrm{O}(n, J)$, the subgroup of $\mathrm{GL}(n, \mathbb{R})$ preserving $J$. This reduces to the $\mathrm{O}(n, \mathbb{R})$ invariance, when $J$ is isotropic, i.e., $J=I \operatorname{Id}_{n} ; I$ is a positive constant of internal inertia and $\operatorname{Id}_{n}$ is the $n \times n$ identity matrix. Then

$$
T_{\mathrm{int}}^{\mathrm{d}^{\prime} \mathrm{A}}=\frac{I}{2} \operatorname{Tr}\left(\xi \xi^{T}\right) .
$$

The total kinetic energy of the body is given by

$$
T^{\mathrm{d}^{\prime} \mathrm{A}}=\sum_{A=1}^{N} T_{A}^{\mathrm{d}^{\prime} \mathrm{A}}=\frac{1}{2} \sum_{A=1}^{N} M_{A} \operatorname{Tr}\left(v_{A} v_{A}^{T}\right)+\frac{1}{2} \sum_{A=1}^{N} \operatorname{Tr}\left(\xi_{A} J_{A} \xi_{A}^{T}\right) .
$$


Assuming that the body consists of identical structure elements we have that $M_{A}=M, J_{A}=J, A=\overline{1, N}$.

Let us again concentrate on a single element. Its Green and Cauchy deformation tensors are respectively denoted as

$$
G[\varphi]=\varphi^{T} \varphi=G[\varphi]^{T}, \quad C[\varphi]=\varphi^{-1 T} \varphi^{-1}=C[\varphi]^{T},
$$

similarly, for their contravariant inverses we write

$$
\widetilde{G}[\varphi]=\varphi^{-1} \varphi^{-1 T}, \quad \widetilde{C}[\varphi]=\varphi \varphi^{T} .
$$

Spatial and material transformations are respectively given by left and right regular translations:

$$
\varphi \mapsto L_{A}(\varphi)=A \varphi, \quad \varphi \mapsto R_{A}(\varphi)=\varphi A
$$

for any fixed $A \in \mathrm{GL}(n, \mathbb{R})$. When $A \in \mathrm{O}(n, \mathbb{R})$, then obviously

$$
G[A \varphi]=G[\varphi], \quad C[\varphi A]=C[\varphi],
$$

and for the general $A \in \mathrm{GL}(n, \mathbb{R})$

$$
G[\varphi A]=A^{T} G[\varphi] A, \quad C[A \varphi]=A^{-1 T} C[\varphi] A^{-1} .
$$

There is no concise formula for $G[A \varphi], C[\varphi A]$ if $A$ is not orthogonal (does not belong to $\mathrm{O}(n, \mathbb{R}))$.

Deformation invariants are scalar functions $f: \operatorname{GL}(n, \mathbb{R}) \rightarrow \mathbb{R}$ invariant under (14) for orthogonal translations

$$
f(A \varphi B)=f(\varphi)
$$

for any $A, B \in \mathrm{O}(n, \mathbb{R})$. There are $n$ basic invariants through which all other ones may be expressed. Various choices are possible, e.g., the following frequently used

$$
\mathcal{K}_{a}[\varphi]=\operatorname{Tr}\left(G[\varphi]^{a}\right)=\operatorname{Tr}\left(C[\varphi]^{-a}\right), \quad a=\overline{1, n},
$$

eigenvalues $\lambda_{a}[\varphi]$ of $G[\varphi]$,

$$
\operatorname{det}\left(G[\varphi]-\lambda[\varphi] I_{n}\right)=0,
$$

or coefficients $I_{p}[\varphi]$ of the eigenequation

$$
\operatorname{det}\left(G[\varphi]-\lambda I_{n}\right)=\sum_{k=0}^{n}(-1)^{k} I_{n-k}[\varphi] \lambda^{k} ;
$$

obviously, $I_{0}=1$ is standard. Geometrically speaking, deformation invariants are functions on the manifold of double cosets

$$
\operatorname{Inv}:=\mathrm{O}(n, \mathbb{R}) \backslash \mathrm{GL}(n, \mathbb{R}) / \mathrm{O}(n, \mathbb{R}) .
$$


Deformation invariants are used when constructing potential energy models for a single affine body. When dealing with the system of such bodies we need some basic scalars assigned to pairs of internal configurations. In analogy to Green and Cauchy deformation tensors for any pair $\psi, \varphi \in \mathrm{GL}(n, \mathbb{R})$ we define the quantities

$$
G[\psi, \varphi]:=\psi^{T} \varphi, \quad C[\psi, \varphi]:=\varphi^{-1 T} \psi^{-1} .
$$

Obviously,

$$
G[\psi, \psi]=G[\psi], \quad C[\psi, \psi]=C[\psi],
$$

i.e., the above mutual deformation tensors reduce then to the usual ones.

But one can also define another mutual quantities, namely,

$$
\Gamma[\psi, \varphi]:=\psi^{-1} \varphi, \quad \Sigma[\psi, \varphi]:=\varphi \psi^{-1} .
$$

For orthogonal matrices they reduce to the previous ones,

$$
\psi, \varphi \in \mathrm{O}(n, \mathbb{R}) \Rightarrow \Gamma[\psi, \varphi]=G[\psi, \varphi], \quad \Sigma[\psi, \varphi]=C[\psi, \varphi] .
$$

Obviously, $\Gamma[\psi, \varphi], \Sigma[\psi, \varphi]$ are exactly group-theoretical counterparts of the displacement vector in translational degrees of freedom. Indeed, interpreting $\mathbb{R}^{n}$ as an Abelian group under addition of vectors, we immediately notice that the prescription (22) in the non-Abelian multiplicative matrix group $\operatorname{GL}(n, \mathbb{R})$ has exactly the same group meaning as $u=y-w$ in $\mathbb{R}^{n}$.

It is clear that for any $A \in \mathrm{O}(n, \mathbb{R})$

$$
G[A \psi, A \varphi]=G[\psi, \varphi], \quad C[\psi A, \varphi A]=C[\psi, \varphi],
$$

i.e., they are respectively invariant under spatial and material isometries. For the general $A \in \operatorname{GL}(n, \mathbb{R})$ we have

$$
G[\psi A, \varphi A]=A^{T} G[\psi, \varphi] A, \quad C[A \psi, A \varphi]=A^{-1 T} C[\psi, \varphi] A^{-1} .
$$

And just as previously there is no concise expression for $G[A \psi, A \varphi], C[\psi A, \varphi A]$ if $A$ is non-orthogonal.

Transformation rules for $\Gamma, \Sigma$ have another form. Namely, for any $A \in$ $\operatorname{GL}(n, \mathbb{R})$ we have

$$
\begin{aligned}
\Gamma[A \psi, A \varphi]=\Gamma[\psi, \varphi], & \Sigma[A \psi, A \varphi] & =A \Sigma[\psi, \varphi] A^{-1}, \\
\Gamma[\psi A, \varphi A]=A^{-1} \Gamma[\psi, \varphi] A, & \Sigma[\psi A, \varphi A] & =\Sigma[\psi, \varphi] .
\end{aligned}
$$

Therefore, $\Gamma$ is invariant under spatial affine transformations and suffers the inverse adjoint rule under material affine transformations. And conversely, $\Sigma$ transforms according to the adjoint rule under spatial affine mappings and is affinely invariant under material transformations.

The quantities $G[\psi, \varphi], C[\psi, \varphi], \Gamma[\psi, \varphi], \Sigma[\psi, \varphi]$, give rise to scalars which may be used as arguments of the potential energy terms. Typical scalars of this type are, in analogy to (18), given by

$$
\mathcal{K}_{a}[\psi, \varphi]=\operatorname{Tr}\left(G[\psi, \varphi]^{a}\right)=\operatorname{Tr}\left(C[\psi, \varphi]^{-a}\right), \quad a=\overline{1, n} .
$$


Just as in the case of deformation invariants, these scalars are invariant under spatial and materia rotations (left and right regular translations of $\varphi, \psi$ by orthogonal matrices):

$$
\mathcal{K}_{a}[A \psi B, A \varphi B]=\mathcal{K}_{a}[\psi, \varphi], \quad A, B \in \mathrm{O}(n, \mathbb{R}) .
$$

In analogy to (19), (20) one can also use solutions of the eigenequation for $G[\psi, \varphi]$ (or $C[\psi, \varphi]$ ), or coefficients in the eigenequation as basic invariants. Another kind of invariants is built of $\Gamma, \Sigma$-objects, e.g.,

$$
\mathcal{M}_{a}[\psi, \varphi]=\operatorname{Tr}\left(\Gamma[\psi, \varphi]^{a}\right)=\operatorname{Tr}\left(\Sigma[\psi, \varphi]^{a}\right)
$$

like in (18), or, according to the $\lambda_{a^{-}}, I_{a^{-}}$schemes like in (19), (20). These objects are invariant under all affine spatial and material transformations, i.e.,

$$
\mathcal{M}_{a}[A \psi B, A \varphi B]=\mathcal{M}_{a}[\psi, \varphi], \quad A, B \in \mathrm{O}(n, \mathbb{R})
$$

for any $A, B \in \mathrm{GL}(n, \mathbb{R})$. These scalars measures of the "distance" between internal configurations are affinely invariant. Unlike this, the measures $\mathcal{K}_{a}$ are only orthogonally invariant, so they are usual Euclidean distances.

Let us remind that in many problems it is convenient to use deformation measures which vanish in the non-deformed state, e.g., Lagrange and Euler deformation tensors:

$$
E[\varphi]=\frac{1}{2}(G[\varphi]-I), \quad e[\varphi]=\frac{1}{2}(I-C[\varphi]) .
$$

By analogy, it may be convenient to replace the mutual tensors $G[\psi, \varphi], C[\psi, \varphi]$, $\Gamma[\psi, \varphi], \Sigma[\psi, \varphi]$ by

$$
\begin{aligned}
E[\psi, \varphi]=\frac{1}{2}(G[\varphi]-I), & e[\psi, \varphi] & =\frac{1}{2}(I-C[\varphi]), \\
\gamma[\psi, \varphi]=\Gamma[\psi, \varphi]-I, & \sigma[\psi, \varphi] & =\Sigma[\psi, \varphi]-I .
\end{aligned}
$$

Another possibility is to use the exponential representation of matrices $G, C$, $\Gamma, \Sigma$. It may be also convenient to use invariants built of them according to the schemes like (18), (30) or (19), (20), etc. Obviously, such invariants are functionally dependent on the previous ones.

Affine velocity, i.e., Eringen's "gyration" 2] respectively in the spatial and co-moving representations is given by

$$
\Omega=\frac{d \varphi}{d t} \varphi^{-1}, \quad \widehat{\Omega}=\varphi^{-1} \frac{d \varphi}{d t} .
$$

Spatial and material transformations (14) act on the above quantities as follows:

$$
\begin{array}{ll}
L_{A}: & : \quad \Omega \mapsto A \Omega A^{-1}, \quad \widehat{\Omega} \mapsto \widehat{\Omega}, \\
R_{A}: & \Omega \mapsto \Omega, \quad \widehat{\Omega} \mapsto A^{-1} \widehat{\Omega} A .
\end{array}
$$


When the motion is metrically rigid, i.e., permanently $\varphi \in \mathrm{O}(n, \mathbb{R})$, then

$$
\Omega=-\Omega^{T}, \quad \widehat{\Omega}=-\widehat{\Omega}^{T}
$$

and these skew-symmetric objets reduce to the usual angular velocity, respectively in the spatial and co-moving representations. In some formulas it is convenient to use also the co-moving representation of translational velocity. It is given by

$$
\widehat{v}=\varphi^{-1} v
$$

Gyroscopic constraints may be described in anholonomic terms simply by stating that $\Omega$ is skew-symmetric (and so is $\widehat{\Omega}$ then),

$$
\Omega+\Omega^{T}=0 .
$$

By analogy, constraints of the purely deformative rotationless motion may be defined by the demand of permanently symmetric $\Omega$,

$$
\Omega-\Omega^{T}=0 .
$$

Let us observe that the materially rotationless constraints

$$
\widehat{\Omega}-\widehat{\Omega}^{T}=0
$$

are non-equivalent to the above spatially rotationless ones.

There is an interesting novelty now. Namely, rotationless constraints are essentially non-holonomic. The point is that the subspace of symmetric matrices is not a commutator Lie subalgebra.

Canonical momenta, i.e., dual objects of velocities (linear functions of them), $p, \pi$ are elements of $\mathbb{R}^{n}, \mathrm{~L}(n, \mathbb{R})$ respectively, and their pairing with velocities is given by

$$
\langle p, v\rangle=p^{T} v=\operatorname{Tr}\left(p v^{T}\right), \quad\langle\pi, \xi\rangle=\operatorname{Tr}(\pi \xi) .
$$

The co-moving representation of $p$ is given by

$$
\widehat{p}=\varphi^{T} p,
$$

and obviously, with this convention

$$
\langle p, v\rangle=p^{T} v=\widehat{p}^{T} \widehat{v}=\langle\widehat{p}, \widehat{v}\rangle .
$$

It is convenient to introduce non-holonomic canonical momenta conjugate to $\Omega, \widehat{\Omega}$, namely,

$$
\Sigma:=\varphi \pi, \quad \widehat{\Sigma}:=\pi \varphi .
$$

Obviously,

$$
\langle\Sigma, \Omega\rangle=\operatorname{Tr}(\Sigma \Omega)=\operatorname{Tr}(\widehat{\Sigma} \widehat{\Omega})=\langle\widehat{\Sigma}, \widehat{\Omega}\rangle .
$$

Transformation rules for $\Sigma, \widehat{\Sigma}$ are identical with (36), (37):

$$
\begin{array}{ll}
L_{A}: & \Sigma \mapsto A \Sigma A^{-1}, \quad \widehat{\Sigma} \mapsto \widehat{\Sigma}, \\
R_{A}: & \Sigma \mapsto \Sigma, \quad \widehat{\Sigma} \mapsto A^{-1} \widehat{\Sigma} A .
\end{array}
$$


The components of $\Sigma, \widehat{\Sigma}$ are respectively Hamiltonian generators of spatial and material affine transformations (14). Their doubled skew-symmetric parts

$$
S=\Sigma-\Sigma^{T}=-S, \quad V=\widehat{\Sigma}-\widehat{\Sigma}^{T}=-V
$$

are, respectively, Hamiltonian generators of spatial and material rotations of internal degrees of freedom.

The relationship between Hamiltonian quantities like $p, \widehat{p}, \Sigma, \widehat{\Sigma}$ and kinematical ones like $v, \widehat{v}, \Omega, \widehat{\Omega}$ may be established only on the basis of some particular model, when Lagrangian $L$ is fixed. In potential models $L=T-\mathcal{V}$ with the potential $\mathcal{V}$ depending only on the configuration $(x, \varphi) \in \mathbb{R}^{n} \times \mathrm{GL}(n, \mathbb{R})$, Legendre transformation has the following form:

$$
p_{i}=\frac{\partial L}{\partial v^{i}}=\frac{\partial T}{\partial v^{i}}, \quad \pi_{i}^{A}=\frac{\partial L}{\partial \xi^{i} A}=\frac{\partial T}{\partial \xi^{i} A} .
$$

Assuming the d'Alembert model of the kinetic energy (6) we obtain that

$$
p=M v, \quad \pi=J \xi^{T},
$$

and the corresponding expression for the kinetic Hamiltonian

$$
\mathcal{T}=\mathcal{T}_{\text {tr }}+\mathcal{T}_{\text {int }}=\frac{1}{2 M} \operatorname{Tr}\left(p p^{T}\right)+\frac{1}{2} \operatorname{Tr}\left(\pi^{T} J^{-1} \pi\right) .
$$

For Hamiltonian systems $H=\mathcal{T}+\mathcal{V}(\mathcal{V}$ denoting the potential energy) equations of motion may be effectively analyzed in terms of Poisson brackets and canonical Hamilton equations,

$$
\frac{d F}{d t}=\{F, H\},
$$

where $F$ runs over some maximal system of functionally independent phasespace functions.

\section{Dynamical models. Affine invariance prob- lems. Realistic questions, academic questions, and pure fantasy}

For the system of affine bodies Lagrangian has the form:

$$
L=T-\mathcal{V},
$$

where the kinetic energy is obtained by summation of individual kinetic energies like in (11),

$$
T=\sum_{A=1}^{N} T_{A}=T_{\mathrm{tr}}+T_{\mathrm{int}}=\sum_{A=1}^{N}\left(T_{\mathrm{tr}}\right)_{A}+\sum_{A=1}^{N}\left(T_{\mathrm{int}}\right)_{A} .
$$


The potential energy in typical situations consists of two main terms, the oneand two-body potentials,

$$
\mathcal{V}=\mathcal{V}^{(1)}+\mathcal{V}^{(2)}
$$

It is known that in realistic problems it is usually less then $10 \%$ of energy that could be assigned to three-body and higher multibody interactions. $\mathcal{V}^{(1)}$ is the sum of terms depending on individual elements,

$$
\mathcal{V}^{(1)}\left(\ldots ; x_{A}, \varphi_{A} ; \ldots\right)=\sum_{B=1}^{N} \mathcal{V}^{(1)}{ }_{B}\left(x_{B}, \varphi_{B}\right)
$$

The over-simplified models where $\mathcal{V}^{(1)}{ }_{B}$ splits into the sum of translational and internal parts,

$$
\mathcal{V}^{(1)}{ }_{B}\left(x_{B}, \varphi_{B}\right)=\mathcal{V}_{\operatorname{tr}}^{(1)}{ }_{B}\left(x_{B}\right)+\mathcal{V}_{\text {int }}^{(1)}\left(\varphi_{B}\right)
$$

are not very realistic, nevertheless, they provide some so-to-speak zeroth-order approximation. When the elements are identical, all $\mathcal{V}^{(1)}{ }_{B}$ have the same functional form.

The binary term has the usual form,

$$
\mathcal{V}^{(2)}\left(\ldots ; x_{A}, \varphi_{A} ; \ldots\right)=\frac{1}{2} \sum_{K, L=1}^{N} \mathcal{V}_{K L}^{(2)}\left(x_{K}, \varphi_{K} ; x_{L}, \varphi_{L}\right)
$$

And again the simplest, although rather academic models are those with the separated dependence of $\mathcal{V}^{(2)}$ on translational and internal variables,

$$
\mathcal{V}^{(2)}{ }_{K L}\left(x_{K}, \varphi_{K} ; x_{L}, \varphi_{L}\right)=\mathcal{V}_{\mathrm{tr}}^{(2)} K L\left(x_{K}, x_{L}\right)+\mathcal{V}_{\text {int } K L}^{(2)}\left(\varphi_{K}, \varphi_{L}\right)
$$

Mutual interactions should be translationally invariant, i.e., $\mathcal{V}^{(2)} K L$ depend on $x_{K}, x_{L}$ through $\overrightarrow{x_{K} x_{L}}=x_{L}-x_{K}$. Isotropy of the physical space implies that the radius-vectors $x_{L}-x_{K}$ enter $\mathcal{V}^{(2)}{ }_{K L}$ only through their lengths $\left\|x_{L}-x_{K}\right\|$. There is some more discussion concerning the dependence of $\mathcal{V}^{(2)} K L$ on internal degrees of freedom. Isotropy of the physical space implies that $\mathcal{V}_{\text {int } K L}^{(2)}$ should depend on $\varphi_{K}, \varphi_{L}$ only through the mutual tensors $G\left[\varphi_{K}, \varphi_{L}\right], \Gamma\left[\varphi_{K}, \varphi_{L}\right]$, thus,

$$
\mathcal{V}^{(2)}{ }_{K L}\left(x_{k}, \varphi_{k} ; x_{L}, \varphi_{L}\right)=f_{K L}\left(\left\|x_{L}-x_{K}\right\|, G\left[\varphi_{K}, \varphi_{L}\right], \Gamma\left[\varphi_{K}, \varphi_{L}\right]\right)
$$

and obviously for the body consisting of identical elements there is no dependence on $K, L ; f_{K L}=f$ for some fixed $f$. And if the dynamics is to be invariant also under simultaneous material rotations, then at the same time, $\mathcal{V}^{(2)}{ }_{K L}$ must depend on internal configurations only through $C\left[\varphi_{K}, \varphi_{L}\right], \Sigma\left[\varphi_{K}, \varphi_{L}\right]$. But this means that $\mathcal{V}^{(2)} K_{L}$ is algebraically built of the mutual invariants, e.g., chosen as $\mathcal{K}_{a}\left[\varphi_{K}, \varphi_{L}\right], \mathcal{M}_{a}\left[\varphi_{K}, \varphi_{L}\right]$

$$
\mathcal{V}^{(2)}{ }_{K L}\left(x_{K}, \varphi_{K} ; x_{L}, \varphi_{L}\right)=f_{K L}\left(\left\|x_{L}-x_{K}\right\|, \mathcal{K}\left[\varphi_{K}, \varphi_{L}\right], \mathcal{M}\left[\varphi_{K}, \varphi_{L}\right]\right) .
$$

In the last formula, $\mathcal{K}, \mathcal{M}$ are abbreviations for the systems $\mathcal{K}_{a}, \mathcal{M}_{a}, a=\overline{1, n}$. 
In our model, geometry of degrees of freedom and kinematics is ruled by the affine group. On the other hand, the dynamics is not invariant either under spatial or material affine transformations (14). The spatial metric tensor and the inertial moment $J$ break the affine symmetry and restrict it to the Euclidean one in the physical space and to $\mathrm{O}(n, J)$ in the material space. What concerns potential energy of mutual interactions (63) it is clear that the vector norm $\left\|x_{L}-x_{K}\right\|$ and transposition-dependent invariants $\mathcal{K}\left[\varphi_{K}, \varphi_{L}\right]$ also restrict the spatial symmetry to $\mathrm{O}(n, \mathbb{R})$. But it is well-known that particularly interesting models and successful analytical procedures appear when the group of dynamical symmetries (symmetries of Lagrangian) coincides with the kinematical group, or at least, when it is as large a subgroup as possible. The questions arise as to the formal possibility and physical usefulness of affinely-invariant models. For a single affinely-rigid body such models are in a sense possible 9, 10. Their physical usefulness is not yet decided, although there are some arguments supporting it. Namely, it is quite possible that in complex media with a complicated net of internal interactions a single element is more sensitive to its material surrounding than to the "true" metric tensor (produced, according to General Relativity by the gravitational field as its "vacuum" nonexcited state). The more so such a mechanism works in defect theory. Let us also mention the concept of effective mass [13, 14] in crystals, where the kinetic energy of electrons is not based on the "true" metric, but on the effective tensor produced by the material surroundings. There are nice mathematical models of the kinetic energy of a single affine body with the kinetic energy based on the Cauchy tensor used as a metric. There are also some physical arguments supporting such a hypothesis [9, 10].

The material affine invariance may seem perhaps more natural because there exist models of continua based on very rich material symmetry. As mentioned, this is Arnold description of the ideal incompressible fluid. It is based on infinitedimensional group of volume-preserving diffeomorphism. They act on the right, i.e., as material transformations. In any case, finite-dimensional geodetic models of small grains or suspensions with kinetic energies materially invariant under $\mathrm{SL}(n, \mathbb{R})$ may be considered as on over-simplified, drastically discretized version of the Arnold model.

Obviously, apparently the Euclidean invariance of the kinetic energy of single elements and of mutual potential elements seems to be firmly established. But nevertheless it may be a superficial illusion and affine invariance should be at least admitted to consideration. When one deals with a highly condensed matter and with very small structure elements, e.g., in the nanoscale, then the complicated structure of interactions may result in quite unexpected results. Namely that some hypothetic phenomenological models based only on some symmetry guiding hints may be so realistic as (or even more realistic) than ones based on apparently careful structural "derivation". It is so because "derivation" in strongly interacting structured media always neglects a lot of factors and the collective effective phenomena are better described by phenomenological models derived on the basis of well-established invariance assumptions. It is so, e.g., in such complicated structures like atomic nuclei. In any case, symmetry principles 
are then rather reliable guiding hints.

For a single affine body non-trivial potentials of internal degrees of freedom are never affinely invariant. Only constant functions on $\mathrm{GL}(n, \mathbb{R})$ may be so. The same is true for one-particle external potentials of the system. As we have just seen, for the purely mutual interactions, the binary potentials of internal degrees of freedom admit affine invariants as arguments. As we shall see, at least formally, the same is true for translational degrees of freedom. We shall go back to this problem later on and concentrate now on the kinetic energy terms.

There exist at least academically interesting kinetic energies for single affine bodies, and obviously, for their systems as well (because, unlike potentials, the kinetic energy is additive).

Of course, the usual d'Alembert model (6) of $T_{\text {int }}$ is isotropic in the physical space and affinely invariant under material affine transformations. This is also explicitly visualized by its another equivalent representations,

$$
T_{\mathrm{tr}}^{\text {is }-\mathrm{af}}=\frac{M}{2} \operatorname{Tr}\left(v v^{T}\right)=\frac{M}{2} v^{T} v=\frac{M}{2} \widehat{v}^{T} G[\varphi] \widehat{v}=\frac{M}{2} \operatorname{Tr}\left(\widehat{v}^{T} G[\varphi]\right) .
$$

And the same holds for any of the structural element, i.e., for any $T_{A}$. Obviously, $v, \widehat{v}, G$ must be then replaced by the corresponding $v_{A}, \widehat{v}_{A}, G_{A}=G\left[\varphi_{A}\right]$.

The labels "tr", "is", "af" in (64) refer respectively to "translational part", "isometry invariant in the physical space" (thus, written on the left-hand-side: left-invariant), and "affinely invariant in the material space of a single element" (thus, written on the right-hand-side: right-invariant).

From the purely academic point of view one can also think about $T_{\mathrm{tr}}^{\mathrm{af}-\mathrm{is}}$, the model affinely invariant in the physical space and isometry invariant in the material space (respectively, left and right invariance in $\operatorname{GL}(n, \mathbb{R})$ ). It will have the form:

$$
T_{\mathrm{tr}}^{\mathrm{af}-\mathrm{is}}=\frac{M}{2} \widehat{v}^{T} \widehat{v}=\frac{M}{2} \operatorname{Tr}\left(\widehat{v} \widehat{v}^{T}\right)=\frac{M}{2} v^{T} C[\varphi] v=\frac{M}{2} \operatorname{Tr}\left(v v^{T} C[\varphi]\right) .
$$

The Cauchy deformation tensor is now used as the "metric" of the physical space.

Let us observe, there is no possibility to obtain translational kinetic energy which would be affinely invariant in both the spatial and material sense, at least if we do not try some extremely exotic things. The reason is that the affine group is non-simple in a very special way and does not admit doubly-invariant and non-degenerate twice covariant tensor fields. Let us observe that the trick with the Cauchy tensor substituted instead of the "usual" metric tensor may be as well repeated for the internal part of (6) . This would lead to the following expressions:

$$
T_{\mathrm{tr}}^{\mathrm{af}-\mathrm{J}}=\frac{1}{2} \operatorname{Tr}\left(C[\varphi] \xi J \xi^{T}\right)=\frac{1}{2} \operatorname{Tr}\left(\widehat{\Omega} J \widehat{\Omega}^{T}\right) .
$$

Here the upper-case labels at $T_{\text {int }}$ mean that it is invariant under all spatial affine transformations and under the material group $\mathrm{O}(n, J) \subset \mathrm{GL}(n, \mathbb{R})$ of $J$ preserving material transformations ("material isometries" when interpreting $J$ as kind of the "material metric tensor"). The expression (66) becomes $T_{\mathrm{int}}^{\mathrm{af}-\mathrm{is}}$, 
i.e., materially isotropic in the usual sense when $J$ is spherical, i.e., $J=I \operatorname{Id}_{n}$, where $I$ is the scalar inertial parameter and $\operatorname{Id}_{n}$ is the unit $n \times n$ matrix. Then we have

$$
T_{\mathrm{tr}}^{\mathrm{af}-\mathrm{is}}=\frac{I}{2} \operatorname{Tr}\left(\widehat{\Omega} \widehat{\Omega}^{T}\right)=\frac{I}{2} \operatorname{Tr}\left(C[\varphi] \xi \xi^{T}\right) .
$$

Let us observe that the spatially affine models (65), (66) are constantcoefficient quadratic forms only when expressed in terms of non-holonomic velocities $\widehat{\Omega}$, therefore, the metric tensors underlying them are curved, essentially Riemannian.

One can wonder what would be a possibility symmetric to (66), i.e., affinely invariant in the material sense. In this sense the natural modification of $T_{\mathrm{int}}$ in (6) would be

$$
T_{\mathrm{tr}}^{\mathrm{H}-\mathrm{af}}=\frac{1}{2} \operatorname{Tr}\left(\Omega H \Omega^{T}\right)=\frac{1}{2} \operatorname{Tr}\left(\xi H[\varphi] \xi^{T}\right),
$$

where $H$ is a fixed positively definite matrix, and $H[\varphi]$ is its following $\varphi^{-1}$ transform:

$$
H[\varphi]=\varphi^{-1} H \varphi^{-1 T} .
$$

So, from the point of view of the usual d'Alembert theory $H[\varphi]$ is a strange $\varphi$-dependent although co-moving inertial tensor, whereas its spatial representation is constant. In the usual d'Alembert affine dynamics the co-moving $J$ is constant, whereas its spatial representation $J[\varphi]$ is configuration-dependent, thus, also time-dependent:

$$
J[\varphi]=\varphi J \varphi^{T} .
$$

The above affine models of $T_{\text {int }}$ are very peculiar in the sense that the inertial terms in (66), (68) are factorized into tensor products. (The factors additional to $H, J$ are the physical and material metric tensors. They are apparently absent because we use Cartesian coordinates which analytically reduce the metric tensors to identity matrices.) The most general $T_{\text {int }}$ invariant under spatial and material affine transformations are respectively given by

$$
\begin{aligned}
T_{\mathrm{int}}^{\mathrm{l}-\mathrm{af}} & =\frac{1}{2} \mathcal{L}^{B}{ }_{A}{ }_{C}{ }_{C} \widehat{\Omega}^{A}{ }_{B} \widehat{\Omega}^{C}{ }_{D}, \\
T_{\mathrm{int}}^{\mathrm{r}-\mathrm{af}} & =\frac{1}{2} \mathcal{R}^{j}{ }_{i{ }_{k}{ }_{k} \Omega^{i}{ }_{j} \Omega^{k}{ }_{l},}
\end{aligned}
$$

where $\mathcal{L}, \mathcal{R}$ are constant and symmetric in their bi-indices (under exchanging mutually the first and second pairs of indices).

The only model of $T_{\text {int }}$ invariant simultaneously under spatial and material affine transformations (left and right regular translations in $\operatorname{GL}(n, \mathbb{R})$ ) has the form:

$$
T_{\mathrm{int}}^{\mathrm{af}-\mathrm{af}}=\frac{A}{2} \operatorname{Tr}\left(\Omega^{2}\right)+\frac{B}{2}(\operatorname{Tr} \Omega)^{2}=\frac{A}{2} \operatorname{Tr}\left(\widehat{\Omega}^{2}\right)+\frac{B}{2}(\operatorname{Tr} \widehat{\Omega})^{2},
$$

where $A, B$ are inertial constants.

This affine-affine model of $T_{\text {int }}$ is never positively-definite. One can show that this "failure" is non-embarrassing, moreover, it may be just profitable [9, 10]. 
It was told above that the translational part $T_{\mathrm{tr}}$ is never simultaneously left (spatial) and right (material) affinely invariant. The highest available symmetries of $T_{\mathrm{tr}}$ are $T_{\mathrm{tr}}^{\mathrm{is}-\mathrm{af}}$ and $T_{\mathrm{tr}}^{\mathrm{af}-\mathrm{is}}$ (64), (65). This raises the question as to the internal counterparts $T_{\text {int }}^{\text {is-af }}$ and $T_{\text {int }}^{\text {af }- \text { is }}$. One can easily show they are given by

$$
\begin{aligned}
T_{\mathrm{int}}^{\mathrm{is}-\mathrm{af}} & =\frac{I}{2} \operatorname{Tr}\left(\Omega^{T} \Omega\right)+T_{\mathrm{int}}^{\mathrm{af}-\mathrm{af}}, \\
T_{\mathrm{int}}^{\mathrm{af}-\mathrm{is}} & =\frac{I}{2} \operatorname{Tr}\left(\widehat{\Omega}^{T} \widehat{\Omega}\right)+T_{\mathrm{int}}^{\mathrm{af}-\mathrm{af}},
\end{aligned}
$$

where $I$ is an additional inertial constant. In the special case of the metricallyrigid (gyroscopic) motion, the first terms in (74), (75) coincide. In general this is not the case. Therefore, if gyroscopic constraints are imposed (no deformations), (74) and (75) coincide, and one obtains the spherical rigid body with the scalar inertial momentum $(I-A)$.

Unlike (73), expressions (74), (75) may be positively definite and they are so in some open range of triples $(I, A, B)$. And at the same time they continue to have the main advantages and the general structure of (73), both on the level of theoretical analysis and practical calculations.

Obviously, everything said above concerns directly a single affinely-deformable element, nevertheless, just as in the d'Alembert model, applies also immediately to the total system, because the modified kinetic energies are additive. One should only use explicitly the label $K$ referring to structural elements.

And now let us go back to the problem of potential energy. As mentioned, the external one-particle potential $\mathcal{V}^{(1)}$ cannot be affinely invariant (only constant functions may be so). The formula (63) for the doubly isotropic binary potential $\mathcal{V}_{K L}^{(2)}$ seems to suggest something similar for the dynamics of mutual interactions. However, things are not so simple and one can try to find some modifications towards the affine invariance, just as it was done in the case of kinetic energy models. Some at least formally admissible suggestion may be easily formulated.

Let us fix some pair of structural elements labelled by $(K, L)$. Internal configurations $\varphi_{K}, \varphi_{L} \in \mathrm{GL}(n, \mathbb{R})$ give rise to the Cauchy tensors $C\left[\varphi_{K}\right], C\left[\varphi_{L}\right]$. In our considerations above we were faced with the idea of using $C[\varphi]$ as a kind of spatial "metric tensor" underlying affinely-invariant kinetic energies of single elements. Let us now introduce the objects

$$
C\left[\varphi_{K}, \varphi_{L}\right]=\frac{1}{2}\left(C\left[\varphi_{K}\right]+C\left[\varphi_{L}\right]\right) .
$$

It is symmetric in the labels $K, L$ and positively definite. This motivates the temptation to use it as a "metric tensor" underlying some modified "distance" between $x_{K}$ and $x_{L}$, namely,

$$
\begin{aligned}
\mathcal{D}\left[x_{K}, \varphi_{K} ; x_{L}, \varphi_{L}\right] & =\sqrt{\left(x_{K}-x_{L}\right)^{T} C\left[\varphi_{K}, \varphi_{L}\right]\left(x_{K}-x_{L}\right)} \\
& =\sqrt{\operatorname{Tr}\left(C\left[\varphi_{K}, \varphi_{L}\right]\left(x_{K}-x_{L}\right)\left(x_{K}-x_{L}\right)^{T}\right)} .
\end{aligned}
$$


Obviously, the transformation rule (16) implies that the above prescription is invariant under the spatial action of $\mathrm{GL}(n, \mathbb{R})$ :

$$
\mathcal{D}\left[A x_{K}, A \varphi_{K} ; A x_{L}, A \varphi_{L}\right]=\mathcal{D}\left[x_{K}, \varphi_{K} ; x_{L}, \varphi_{L}\right]
$$

for any $A \in \mathrm{GL}(n, \mathbb{R})$. This is a rather curious affinely-invariant "distance". And now we can modify (63) by introducing to it this new distance-like argument in addition to the usual one:

$$
\left\|x_{K}-x_{L}\right\|=\sqrt{\left(x_{K}-x_{L}\right)^{T}\left(x_{K}-x_{L}\right)}=\sqrt{\operatorname{Tr}\left(\left(x_{K}-x_{L}\right)\left(x_{K}-x_{L}\right)^{T}\right)} .
$$

So, finally, instead of (63) we have

$$
\begin{aligned}
& \mathcal{V}_{K L}^{(2)}\left(x_{K}, \varphi_{K} ; x_{L}, \varphi_{L}\right)= \\
& =f_{K L}\left(\left\|x_{K}-x_{L}\right\|, \mathcal{D}\left[x_{K}, \varphi_{K} ; x_{L}, \varphi_{L}\right], \mathcal{K}\left[\varphi_{K}, \varphi_{L}\right], \mathcal{M}\left[\varphi_{K}, \varphi_{L}\right]\right),
\end{aligned}
$$

where in realistic situations all $f_{K L}$ coincide with some fixed $f$. It is seen that $\mathcal{V}_{K L}^{(2)}$ depends on its configuration arguments through the system of four scalar quantities. Two of them, namely, $\left\|x_{K}-x_{L}\right\|$ and $\mathcal{K}\left[\varphi_{K}, \varphi_{L}\right]$ are invariants of the rotation group $\mathrm{O}(n, \mathbb{R})$. The remaining two, $\mathcal{D}\left[x_{K}, \varphi_{K} ; x_{L}, \varphi_{L}\right]$ and $\mathcal{M}\left[\varphi_{K}, \varphi_{L}\right]$, are invariant under the total linear group $\operatorname{GL}(n, \mathbb{R})$. One can expect that the dependence of $\mathcal{V}^{(2)}$ on the latter two scalars is a highly symmetric, affine background of mutual interactions between constituents of the body. Further on, this high affine symmetry is broken and reduced to the orthogonal one $\mathrm{O}(n, \mathbb{R})$ by the arguments $\left\|x_{K}-x_{L}\right\|, \mathcal{K}\left[\varphi_{K}, \varphi_{L}\right]$. This may happen in such a way that $\mathcal{V}^{(2)}$ is a sum of some purely affine term dependent only on $\mathcal{D}, \mathcal{M}$ and on an appropriate symmetry-restricting metrical term built of $\|\cdot\|$ and $\mathcal{K}$.

It is a very interesting question whether the binary purely affine models

$$
\mathcal{V}_{K L}^{(2) \mathrm{af}}=f_{K L}(\mathcal{D}, \mathcal{M})
$$

may be realistic. The question was not yet touched seriously. Nevertheless, some limitations of applicability of the binary affine paradigm seem to be obvious. In our earlier papers [9, 10] we discussed dynamical models of a single affinely-rigid body, in particular, the purely geodetic models, i.e., ones without potentials. Lagrangian coincides then with the kinetic energies (metric tensors on $\operatorname{GL}(n, \mathbb{R}))$ given by (71) or (72), and first of all, by their special cases like (73), (74), (75). It turns out that for incompressible affine bodies, when the configuration space of internal motion is restricted to $\mathrm{SL}(n, \mathbb{R})$, the purely geodetic affine models predict the existence of an open family of bounded (oscillatory) trajectories within the general solution. However, on the non-restricted $\mathrm{GL}(n, \mathbb{R})$, when the volume changes are admitted, geodetic affine models predict the non-restricted dilatational motion, i.e., unlimited expansion or contraction. This is an evidently non-physical feature of these models. Therefore, at least some dilatations-stabilizing potential $\mathcal{V}_{\text {dil }}(\operatorname{det} \varphi)$ must be assumed. When we 
deal with systems of affine bodies, then it is clear that for an appropriate choice of $f$ in (79) the relative volumes

$$
\operatorname{det} \varphi_{L} / \operatorname{det} \varphi_{K}=\operatorname{det}\left(\varphi_{K}{ }^{-1} \varphi_{L}\right)=\operatorname{det} \Gamma\left[\varphi_{K}, \varphi_{L}\right]
$$

are stabilized in the sense of performing bounded motions. However, no binary potential may stabilize the single volumes $\operatorname{det} \varphi_{K}$ themselves. Their time evolution will be non-bounded although the ratios (80) are bounded functions of time. To prevent this one should introduce some one-body potential term stabilizing (making bounded) the over-all dilatational behaviour,

$$
\mathcal{V}_{\text {dil }}^{(1)}\left(\ldots, \varphi_{A}, \ldots\right)=\sum_{K=1}^{N} \mathcal{V}_{\operatorname{dil} K}^{(1)}\left(\operatorname{det} \varphi_{K}\right)
$$

It is reasonable to assume that all $\mathcal{V}_{\text {dil } K}^{(1)}$ are identical (when the body consists of identical elements),

$$
\mathcal{V}_{\mathrm{dil}}^{(1)}\left(\ldots, \varphi_{A}, \ldots\right)=\sum_{K=1}^{N} f\left(\operatorname{det} \varphi_{K}\right)
$$

When $\mathcal{V}_{K L}^{(2)}$ depend on their arguments in a proper way, so that $\operatorname{det} \Gamma\left[\varphi_{K}, \varphi_{L}\right]$ are bounded functions of time, then in principle it would be sufficient to use $\mathcal{V}_{\text {dil }}^{(1)}$ depending on $\operatorname{det} \varphi_{A}$ for some fixed label $A$ only. If such $\mathcal{V}^{(1)}\left(\operatorname{det} \varphi_{A}\right)$ stabilizes $\operatorname{det} \varphi_{A}$, then automatically all volumes $\operatorname{det} \varphi_{K}$ will be stabilized by $\mathcal{V}^{(2)}$. But of course such a choice of the shape of $\mathcal{V}$ would not be either aesthetic or reasonable.

\section{General quantization ideas}

There is a direct logical chain from the atomic and molecular structure to macroscopic properties, constitutive laws and material engineering. The point is particularly delicate on the nano-level, where one is dealing with a very peculiar convolution of quantum and classical concepts. In any case, quantization is necessary then. Also some quasi-classical and correspondence problems are very relevant for these phenomena.

The first step towards quantization is the classical canonical formalism [15, [11, 8]. One should start from Legendre transformations which for potential systems with Lagrangians $L=T-\mathcal{V}\left(\cdots ; x_{K}, \varphi_{K} ; \cdots\right)$ are given by

$$
p^{K}{ }_{i}=\frac{\partial L}{\partial v^{i}{ }_{K}}=\frac{\partial T}{\partial v^{i} K}, \quad \pi^{K a}{ }_{i}=\frac{\partial L}{\partial \xi_{K^{i}}{ }_{a}}=\frac{\partial T}{\partial \xi_{K^{i}}{ }_{a}}
$$

or, alternatively,

$$
\begin{aligned}
& \widehat{p}^{K}{ }_{a}=\frac{\partial L}{\partial \widehat{v}^{a}{ }_{K}}=\frac{\partial T}{\partial \widehat{v}^{a} K}, \quad \widehat{\Sigma}^{K a}{ }_{b}=\frac{\partial L}{\partial \widehat{\Omega}_{K}^{b}{ }_{a}}=\frac{\partial T}{\partial \widehat{\Omega}_{K}^{b}{ }_{a}}, \\
& \Sigma^{K i}{ }_{j}=\frac{\partial L}{\partial \Omega_{K}{ }_{i}}=\frac{\partial T}{\partial \Omega_{K}{ }_{i}} .
\end{aligned}
$$


Inverting these formulas and substituting them to the energy expression

$$
E=v_{K}^{i} \frac{\partial L}{\partial v^{i} K}+\xi_{K}{ }^{i}{ }_{a} \frac{\partial L}{\partial \xi_{K}^{i}{ }_{a}}-L
$$

one obtains the classical Hamiltonian

$$
H=\mathcal{T}+\mathcal{V}
$$

Let us quote the resulting formulas for the geodetic (kinetic) Hamiltonians $\mathcal{T}$. For the "usual" d'Alembert model (6) we obtain

$$
\mathcal{T}^{\mathrm{d}^{\prime} \mathrm{A}}=\mathcal{T}_{\mathrm{tr}}^{\mathrm{d}^{\prime} \mathrm{A}}+\mathcal{T}_{\text {int }}^{\mathrm{d}^{\prime} \mathrm{A}}=\frac{1}{2 M} \operatorname{Tr}\left(p p^{T}\right)+\frac{1}{2} \operatorname{Tr}\left(\pi^{T} J^{-1} \pi\right) .
$$

This is, as mentioned, the "usual" expression compatible with the d'Alembert principle. Although from some point of view it seems the best-motivated one, in complicated systems with collective modes and strong internal interactions some doubts and just objections may be raised against it. Our idea here was to concentrate on models motivated by symmetry principles, first of all, by affine symmetry. Let us now review Legendre transforms of affine models quoted above.

Of course, the model (64) of translational kinetic energy $T_{\mathrm{tr}}^{\mathrm{is}-\mathrm{af}}$ coincides exactly with $T_{\mathrm{tr}}^{\mathrm{d}^{\prime} \mathrm{A}}$, so we have

$$
\begin{aligned}
\mathcal{T}_{\text {tr }} \text { is-af } & =\frac{1}{2 M} p^{T} p=\frac{1}{2 M} \operatorname{Tr}\left(p p^{T}\right) \\
& =\frac{1}{2 M} \widehat{p}^{T} G[\varphi]^{-1} \widehat{p}=\frac{1}{2 M} \operatorname{Tr}\left(\widehat{p} \widehat{p}^{T} G[\varphi]^{-1}\right),
\end{aligned}
$$

just the first term of (87) written in a few equivalent forms.

The corresponding expression for (65) has the following form:

$$
\begin{aligned}
\mathcal{T}_{\text {tr }}{ }^{\text {af }- \text { is }} & =\frac{1}{2 M} \widehat{p}^{T} \widehat{p}=\frac{1}{2 M} \operatorname{Tr}\left(\widehat{p}^{T}\right) \\
& =\frac{1}{2 M} p^{T} C[\varphi]^{-1} p=\frac{1}{2 M} \operatorname{Tr}\left(p p^{T} C[\varphi]^{-1}\right)
\end{aligned}
$$

Let us now quote the Legendre transforms of affinely-invariant internal kinetic energies. For (66) we obtain

$$
\mathcal{T}_{\text {int }}^{\text {af }-\mathrm{J}}=\frac{1}{2} \operatorname{Tr}\left(C[\varphi]^{-1} \pi^{T} J^{-1} \pi\right)=\frac{1}{2} \operatorname{Tr}\left(\widehat{\Sigma}^{T} J^{-1} \widehat{\Sigma}\right) .
$$

In particular, for the isotropic inertial tensor (67) leads to the expression:

$$
\mathcal{T}_{\text {int }}^{\text {af }- \text { is }}=\frac{1}{2 I} \operatorname{Tr}\left(\widehat{\Sigma}^{T} \widehat{\Sigma}\right)=\frac{1}{2 I} \operatorname{Tr}\left(C[\varphi]^{-1} \pi^{T} \pi\right) .
$$

For the model (68) we obtain

$$
\mathcal{T}_{\text {int }}^{\mathrm{H}-\mathrm{af}}=\frac{1}{2} \operatorname{Tr}\left(\Sigma^{T} H^{-1} \Sigma\right)=\frac{1}{2} \operatorname{Tr}\left(\pi^{T} H[\varphi]^{-1} \pi\right) .
$$


Obviously, the Legendre transforms of the most general affine models (71), (72) have the form:

$$
\begin{aligned}
\mathcal{T}_{\text {int }}^{\text {l-af }} & =\frac{1}{2} \widetilde{\mathcal{L}}^{B}{ }_{A}{ }^{D}{ }_{C} \widehat{\Sigma}^{A}{ }_{B} \widehat{\Sigma}^{C}{ }_{D}, \\
\mathcal{T}_{\text {int }}^{\text {r-af }} & =\frac{1}{2} \widetilde{\mathcal{R}}^{j}{ }_{i k}{ }_{k} \Sigma^{i}{ }_{j} \Sigma^{k}{ }_{l},
\end{aligned}
$$

where the constant bimatrices $\widetilde{\mathcal{L}}, \widetilde{\mathcal{R}}$ are reciprocal to $\mathcal{L}, \mathcal{R}$ respectively.

For the most interesting models (74), (75), including their special doublyaffine case (173) we obtain respectively

$$
\begin{aligned}
& \mathcal{T}_{\text {int }}^{\text {is -af }}=\frac{1}{2 \widetilde{I}} \operatorname{Tr}\left(\Sigma^{T} \Sigma\right)+\frac{1}{2 \widetilde{A}} \operatorname{Tr}\left(\Sigma^{2}\right)+\frac{1}{2 \widetilde{B}}(\operatorname{Tr} \Sigma)^{2}, \\
& \mathcal{T}_{\text {int }}^{\text {af }- \text { is }}=\frac{1}{2 \widetilde{I}} \operatorname{Tr}\left(\widehat{\Sigma}^{T} \widehat{\Sigma}\right)+\frac{1}{2 \widetilde{A}} \operatorname{Tr}\left(\widehat{\Sigma}^{2}\right)+\frac{1}{2 \widetilde{B}}(\operatorname{Tr} \widehat{\Sigma})^{2},
\end{aligned}
$$

where the constants $\widetilde{I}, \widetilde{A}, \widetilde{B}$ are built of $I, A, B$ in the following way:

$$
\widetilde{I}=\frac{1}{I}\left(I^{2}-A^{2}\right), \widetilde{A}=\frac{1}{A}\left(A^{2}-I^{2}\right), \widetilde{B}=-\frac{1}{B}(I+A)(I+A+n B) .
$$

The special affine-affine case (73) corresponds to $I=0$, and then obviously $1 / \widetilde{I}=0$ and the first terms of (95), (96) do vanish. Of course, the second and third terms of (95), (96) are pairwise identical.

These were expressions for various models of the kinetic Hamiltonians for single elements. Obviously, they should be labelled by the index $K=\overline{1, N}$, and the total expression is obtained by a summation over $K$.

Canonical formalism is very convenient and effective in analysis of classical equations of motion. They are represented then in terms of Poisson brackets,

$$
\frac{d F}{d t}=\{F, H\},
$$

where $F$ runs over some maximal functionally independent system of functions. To make use of (98) one must establish the system of basic Poisson brackets for some geometrically distinguished quantities. As a rule, one uses quantities like $\Sigma, \widehat{\Sigma}$, because they are Hamiltonian generators of left and right regular translations in GL $(n, \mathbb{R})$. Therefore, their Poisson brackets are determined by the structure constants of this group (and the mutual Poisson brackets between $\Sigma$ and $\widehat{\Sigma}$-quantities do vanish of course, because the left translations commute with the right ones). Other important Poisson brackets are those between $\Sigma, \widehat{\Sigma}$ quantities and functions depending only on generalized coordinates. Calculating such a bracket is identical with affecting configuration functions by first-order differential operators generating left and right regular translations in $\operatorname{GL}(n, \mathbb{R})$.

However, the main advantage of Hamiltonian methods is that they provide a direct way towards quantization.

Let us remind that the configuration space of our $N$-body system is given by

$$
Q^{N} \simeq Q^{N}{ }_{\text {tr }} \times Q^{N}{ }_{\text {int }} \simeq \mathbb{R}^{n N} \times \mathrm{GL}(n, \mathbb{R})^{N},
$$


i.e., configurations are arrays (5). The manifold $Q^{N}$ is obviously an open subset of the linear space

$$
\mathbb{R}^{n N} \times \mathrm{L}(n, \mathbb{R})^{N} \simeq \mathbb{R}^{n N} \times \mathbb{R}^{n^{2} N} \simeq \mathbb{R}^{n(n+1) N} .
$$

In any one-element configuration space $\mathbb{R}^{n} \times \mathrm{GL}(n, \mathbb{R})$ we are given two distinguished measures. One of them is the Haar measure $\alpha$ invariant under left and right group translations (cf. 9]). The other one is the usual Lebesgue measure $a$ on $\mathbb{R}^{n} \times \mathrm{L}(n, \mathbb{R})$. It is invariant under additive translations. In terms of coordinates

$$
\begin{aligned}
d a(x, \varphi) & =d x^{1} \cdots d x^{n} d \varphi^{1}{ }_{1} \cdots d \varphi^{n}{ }_{n}, \\
d \alpha(x, \varphi) & =(\operatorname{det} \varphi)^{-n-1} d a(x, \varphi) \\
& =(\operatorname{det} \varphi)^{-n-1} d x^{1} \cdots d x^{n} d \varphi^{1}{ }_{1} \cdots d \varphi^{n}{ }_{n} .
\end{aligned}
$$

When we neglect translational motion then the Haar measure $\lambda$ on $\operatorname{GL}(n, \mathbb{R})$ and the Lebesgue measure $l$ on $\mathrm{L}(n, \mathbb{R})$ are used

$$
\begin{aligned}
d l(\varphi) & =d \varphi^{1}{ }_{1} \cdots d \varphi^{n}{ }_{n}, \\
d \lambda(\varphi) & =(\operatorname{det} \varphi)^{-n-1} d l(\varphi)=(\operatorname{det} \varphi)^{-n} d \varphi^{1}{ }_{1} \cdots d \varphi^{n}{ }_{n} .
\end{aligned}
$$

Configuration spaces of the total $N$-element system are endowed with the $N$-told tensor products of these measures, $a^{(N)}, \alpha^{(N)}, l^{(N)}, \lambda^{(N)}$.

The quantized theory is formulated in the following Hilbert spaces:

$$
\mathrm{L}^{2}\left(Q^{N}, \alpha^{(N)}\right), \mathrm{L}^{2}\left(Q^{N}, a^{(N)}\right), \mathrm{L}^{2}\left(\operatorname{GL}(n, \mathbb{R})^{N}, \lambda^{(N)}\right), \mathrm{L}^{2}\left(\operatorname{GL}(n, \mathbb{R})^{N}, l^{(N)}\right) .
$$

Their elements, i.e., wave functions, are complex probability amplitudes of finding the system at a given classical configuration. Classical quantities depending only on configuration variables are represented in these $\mathrm{L}^{2}$-spaces as operators of multiplication by real-valued functions, in particular, by coordinates like $x^{i}, \varphi^{i}{ }_{a}$, etc. According to the general rules of quantum mechanics all other quantities are also represented by Hermitian or formally Hermitian (symmetric in dense domains) operators in these Hilbert spaces. Usually some ordering problems of non-commuting operators appear then. However, in dynamical applications, when Hamiltonian operators are constructed, one deals usually with some special physical quantities of well-defined geometric interpretation. As a rule, they are generators of symmetry groups underlying the problem. In our model they are just the affine spin in both the spatial and co-moving representation, the usual metrical spin and vorticity, etc.

Linear momentum operators in spatial and co-moving representations are given respectively by

$$
\mathbf{p}^{K}{ }_{a}=\frac{\hbar}{i} \frac{\partial}{\partial x^{a}{ }_{K}}, \quad \widehat{\mathbf{p}}^{K}{ }_{a}=\frac{\hbar}{i} \varphi_{K}{ }^{b}{ }_{a} \frac{\partial}{\partial x^{b}{ }_{K}},
$$


where, obviously, $K=\overline{1, N}$ is the "particle" label. These operators are formally Hermitian both in $\mathrm{L}^{2}\left(Q^{N}, \alpha^{(N)}\right)$ and $\mathrm{L}^{2}\left(Q^{N}, a^{(N)}\right)$. The operators

$$
\boldsymbol{\Sigma}_{K}{ }^{a} b=\frac{\hbar}{i} \varphi_{K}{ }^{a}{ }_{c} \frac{\partial}{\partial \varphi_{K}{ }^{b}}, \quad \widehat{\boldsymbol{\Sigma}}_{K}{ }^{a}{ }_{b}=\frac{\hbar}{i} \varphi_{K}{ }^{c} b \frac{\partial}{\partial \varphi_{K}{ }^{c} a}
$$

are formally Hermitian (not literally, they are unbounded as all differential operators) in $\mathrm{L}^{2}\left(Q^{N}, \alpha^{(N)}\right)$ and in $\mathrm{L}^{2}\left(\mathrm{GL}(n, \mathbb{R})^{N}, \lambda^{(N)}\right)$. Therefore, when using these Hilbert spaces we may interpret (104) as operators of affine spin respectively in the spatial and co-moving representations.

Just as in classical theory, $\mathbf{p}^{K}{ }_{a}$ are infinitesimal generators of translations of the $K$-th constituent. Similarly, $\boldsymbol{\Sigma}_{K}{ }^{a} b$ generate spatial affine transformations (rotations and homogeneous deformations) of internal degrees of freedom of the $K$-th "molecule". $\widehat{\boldsymbol{\Sigma}}_{K}^{a} b$ generate material affine transformations of the $K$-th element. Namely, let us consider the operators

$$
\begin{aligned}
& \mathbf{V}_{K}(y):=\exp \left(\frac{i}{\hbar} y^{a} \mathbf{p}^{K}{ }_{a}\right), \quad y \in \mathbb{R}^{n}, \\
& \mathbf{L}_{K}(z):=\exp \left(\frac{i}{\hbar} z^{b}{ }_{a} \boldsymbol{\Sigma}_{K}{ }_{b}{ }_{b}\right), \quad z \in \mathrm{L}(n, \mathbb{R}),
\end{aligned}
$$

where the operator exponent is meant in the usual power-series sense. If this series convergent in the action on some function $\Psi: Q^{N} \rightarrow \mathbb{C}$, then

$$
\begin{aligned}
&\left(\mathbf{V}_{K}(y) \Psi\right)\left(\ldots, x_{A}, \ldots ; \ldots, \varphi_{B}, \ldots\right)= \\
&=\Psi\left(\ldots, x_{A}+y \delta_{A K}, \ldots ; \ldots, \varphi_{B}, \ldots\right) \\
&\left(\mathbf{L}_{K}(z) \Psi\right)\left(\ldots, x_{A}, \ldots ; \ldots, \varphi_{B}, \ldots\right)= \\
&=\Psi\left(\ldots, x_{A} \ldots ; \ldots, \exp \left(z \delta_{K B}\right) \varphi_{B}, \ldots\right)
\end{aligned}
$$

Similar statements may be formulated about the co-moving objects, e.g., defining

$$
\mathbf{R}_{K}(z):=\exp \left(\frac{i}{\hbar} z^{b}{ }_{a} \widehat{\boldsymbol{\Sigma}}_{K}{ }^{a}{ }_{b}\right), \quad z \in \mathrm{L}(n, \mathbb{R}),
$$

we obtain that

$$
\begin{aligned}
\left(\mathbf{R}_{K}(z) \Psi\right)\left(\ldots, x_{A}, \ldots ; \ldots, \varphi_{B}, \ldots\right) & = \\
& =\left(\ldots, x_{A}, \ldots ; \ldots, \varphi_{B} \exp \left(z \delta_{B K}\right), \ldots\right) .
\end{aligned}
$$

One can act separately on all arguments, nevertheless, the special geometric role is played by transformations acting in the same way on all arguments, e.g.,

$$
\begin{aligned}
\mathbf{V}(y) & =\mathbf{V}_{1}(y) \cdots \mathbf{V}_{N}(y) \\
\mathbf{L}(z) & =\mathbf{L}_{1}(z) \cdots \mathbf{L}_{N}(z) \\
\mathbf{R}(z) & =\mathbf{R}_{1}(z) \cdots \mathbf{R}_{N}(z)
\end{aligned}
$$


Their generators are respectively identical with the total linear momentum and the total affine spin in the spatial and co-moving representations,

$$
\mathbf{p}_{a}=\sum_{K=1}^{N} \mathbf{p}^{K}{ }_{a}, \quad \boldsymbol{\Sigma}_{b}^{a}=\sum_{K=1}^{N} \boldsymbol{\Sigma}_{K}{ }_{b}{ }_{b}, \quad \widehat{\boldsymbol{\Sigma}}^{a}{ }_{b}=\sum_{K=1}^{N} \widehat{\boldsymbol{\Sigma}}_{K}{ }_{b}{ }_{b} .
$$

Obviously,

$$
\begin{aligned}
\mathbf{V}(y) & =\exp \left(\frac{i}{\hbar} y^{a} \mathbf{p}_{a}\right) \\
\mathbf{L}(z) & =\exp \left(\frac{i}{\hbar} z^{b}{ }_{a} \boldsymbol{\Sigma}^{a}{ }_{b}\right) \\
\mathbf{R}(z) & =\exp \left(\frac{i}{\hbar} z^{b}{ }_{a} \widehat{\boldsymbol{\Sigma}}^{a}{ }_{b}\right)
\end{aligned}
$$

Obviously, all exponential operators quoted here are unitary in $\mathrm{L}^{2}\left(Q^{N}, \alpha^{(N)}\right)$ or $\mathrm{L}^{2}\left(\mathrm{GL}(n, \mathbb{R})^{N}, \lambda^{(N)}\right)$. However, $\mathbf{L}_{K}, \mathbf{R}_{K}$ are not unitary in $\mathrm{L}^{2}\left(Q^{N}, a^{(N)}\right)$ and $\mathrm{L}^{2}\left(\mathrm{GL}(n, \mathbb{R})^{N}, l^{(N)}\right)$. The reason is that the measures $a, l$ are not invariant under group translations. Also, when working in $\mathrm{L}^{2}\left(Q^{N}, a^{(N)}\right)$ and $\mathrm{L}^{2}\left(\mathrm{GL}(n, \mathbb{R})^{N}, l^{(N)}\right)$, that is admissible, one must modify the definition of the above unitary operators (introducing some multipliers). The generators (104) are not formally Hermitian and to become such they must be modified by some additive corrections:

$$
{ }^{\prime} \boldsymbol{\Sigma}_{K}{ }_{b}{ }_{b}:=\boldsymbol{\Sigma}_{K}{ }^{a}{ }_{b}+\frac{\hbar n}{2 i} \delta_{b}^{a}, \quad \widehat{\boldsymbol{\Sigma}}_{K}{ }^{a}{ }_{b}:=\widehat{\boldsymbol{\Sigma}}_{K}{ }^{a}{ }_{b}+\frac{\hbar n}{2 i} \delta^{a}{ }_{b} .
$$

Let us also quote the formally Hermitian operators

$$
\mathbf{J}_{K}{ }_{b}^{a}=\mathbf{x}_{K}{ }^{a} \mathbf{p}^{K}{ }_{b}+\boldsymbol{\Sigma}_{K}{ }_{b}{ }_{b}=\boldsymbol{\Lambda}_{K}{ }^{a}{ }_{b}+\boldsymbol{\Sigma}_{K}{ }_{b}{ }_{b},
$$

which generate affine transformations acting both on translational and internal degrees of freedom of the $K$-th constituents. $\boldsymbol{\Lambda}_{K}$ and $\boldsymbol{\Sigma}_{K}$ are respectively the translational (orbital) and internal parts. One can also introduce the total quantities

$$
\mathbf{J}_{b}^{a}=\Lambda_{b}^{a}+\boldsymbol{\Sigma}_{b}^{a}
$$

obtained by the $K$-summation.

In analogy to (118), we have that

$$
{ }^{\prime} \boldsymbol{\Lambda}_{K}{ }_{b}{ }_{b}:=\boldsymbol{\Lambda}_{K}{ }^{a}{ }_{b}+\frac{\hbar}{2 i} \delta^{a}{ }_{b} .
$$

Let us observe that for the total quantities built of (118) we have

$$
{ }^{\prime} \boldsymbol{\Sigma}_{b}^{a}=\boldsymbol{\Sigma}_{b}^{a}+\frac{\hbar n N}{2 i} \delta_{b}^{a}, \quad ' \widehat{\boldsymbol{\Sigma}}^{a}{ }_{b}=\widehat{\boldsymbol{\Sigma}}_{b}^{a}+\frac{\hbar n N}{2 i} \delta_{b}^{a} .
$$


and similarly

$$
{ }^{\prime} \boldsymbol{\Lambda}_{b}^{a}=\boldsymbol{\Lambda}_{b}^{a}+\frac{\hbar N}{2 i} \delta_{b}^{a} .
$$

After quantization the canonical momenta $\pi^{K a}{ }_{i}$ conjugate to $\varphi_{K}{ }_{a}{ }_{a}$ become operators:

$$
\mathbf{p}^{K a}{ }_{j}:=\frac{\hbar}{i} \frac{\partial}{\partial \varphi_{K}^{j} a} .
$$

They are formally Hermitian in $\mathrm{L}^{2}\left(Q^{N}, a^{(N)}\right), \mathrm{L}^{2}\left(\mathrm{GL}(n, \mathbb{R})^{N}, l^{(N)}\right)$, but not in $\mathrm{L}^{2}\left(Q^{N}, \alpha^{(N)}\right), \mathrm{L}^{2}\left(\mathrm{GL}(n, \mathbb{R})^{N}, \lambda^{(N)}\right)$, so now the situation is quite opposite to the previous one.

Hamilton operator has the following form:

$$
\mathbf{H}=\mathbf{T}+\mathcal{V},
$$

where $\mathbf{T}$ is the kinetic energy operator and $\mathcal{V}$ is the potential term; usually one does not distinguish graphically between the function $\mathcal{V}$ and the operator $\mathbf{V}$ which multiplies the wave function $\Psi$ by $\mathcal{V}$,

$$
(\mathbf{V} \Psi)(x, \varphi):=\mathcal{V}(x, \varphi) \Psi(x, \varphi) .
$$

In usual structural problems quantum dynamics reduces to the stationary Schrödinger equation, i.e., to the energy following eigenproblem:

$$
\mathbf{H} \Psi=E \Psi .
$$

And finally, we see that constructing the kinetic energy operator $\mathbf{T}$ is a crucial step of the quantization procedure. And this may be done just on the basis of the above classical expressions for kinetic energies in terms of canonical phasespace variables (77), (78), (79), (80), (81), (82), (83), (84), (85), (86). Simply one should algebraically substitute the above operators $\mathbf{p}^{K}{ }_{i}, \widehat{\mathbf{p}}^{K}{ }_{a}, \mathbf{p}^{K a}{ }_{j}, \boldsymbol{\Sigma}^{K a}{ }_{b}$, $\widehat{\boldsymbol{\Sigma}}^{K a}{ }_{b}$ instead of the corresponding classical expressions. Because of the geometric meaning of these quantities as generators of natural transformation groups, there is no problem of ordering of operators. The only point one should be careful with is just the one concerning the measures used in configuration spaces when defining the $\mathrm{L}^{2}$-Hilbert spaces. In affine models the measures $\alpha, \lambda$ are more natural and then one uses the purely differential operators (103), (104). In Hilbert spaces based on $a, l$ we would have to use the modified expressions (118). And conversely, in non-affine d'Alembert models the Lebesgue measures $a, l$ are more natural, because they enable one to use the purely differential operators (124) without any algebraic correction.

The above geometric approach is very convenient. If we tried to calculate the kinetic energy operators as

$$
\mathbf{T}=-\frac{\hbar^{2}}{2} \triangle
$$

where $\triangle$ is the d'Alembert operator based on the metric tensor underlying the classical expression $T$, the calculations would be hopeless and the result completely obscure, non-useful. 
When solving any particular problem one must use coordinates. For our purposes the most convenient choice is that based on the polar and two-polar decompositions:

$$
\varphi=U A=B U=L D R^{-1}=L D R^{T},
$$

where $\varphi \in \mathrm{GL}^{+}(n, \mathbb{R}), U, L, R \in \mathrm{SO}(n, \mathbb{R})$ (orthogonal), $A, B=U A U^{-1}$ are symmetric and positively definite, and $D$ is diagonal and positive.

Green and Cauchy deformation tensors are then expressed as follows:

$$
G=A^{2}=R D^{2} R^{T}, \quad C=B^{-2}=L D^{-2} L^{-1} .
$$

It is convenient to denote

$$
D_{a a}=Q^{a}=\exp \left(q^{a}\right) .
$$

The quantities $Q^{a}, q^{a}$ offer another convenient choices of basic deformation invariants. The Haar and Lebesgue measure $\lambda, l$ are then expressed as

$$
\begin{aligned}
d \lambda(L, D, R) & =\prod_{i \neq j}\left|\operatorname{sh}\left(q^{i}-q^{j}\right)\right| d q^{1} \ldots d q^{n} d \mu(L) d \mu(R), \\
d l(L, D, R) & =\prod_{i \neq j}\left(Q^{i}-Q^{j}\right)\left(Q^{i}+Q^{j}\right) d Q^{1} \ldots d Q^{n} d \mu(L) d \mu(R),
\end{aligned}
$$

where $\mu$ is the Haar measure on $\mathrm{SO}(n, \mathbb{R})$. Performing some partial integrations one can obtain from $\bar{\Psi} \Psi$ probability distributions for some quantities like deformation invariants, orientations of the main axes of Green and Cauchy deformation tensors and also probability distributions for particular values of Green and Cauchy deformation tensors.

\section{Acknowledgements}

The author is greatly indebted to professors Gianfranco Capriz and Paolo Mariano for stimulating discussions in Pisa, Warsaw, and Berlin.

\section{References}

[1] Cosserat, E. and F., 'Theorie des corps deformables', in: Khvol'son, O.D., Traite de physique, translation by Davaux, E. (second ed.), vol. 2, Hermann\&Cie, Paris, 1909, 953-1173.

[2] Eringen, A.C., 'Mechanics of Micromorphic Continua', in: Proceedings of the IUTAM Symposium on Mechanics of Generalized Continua, Freudenstadt and Stuttgart, 1967, editor: Kröner, E., vol. 18, Springer, BerlinHeidelberg-New York, 1968, 18-33.

[3] Capriz, G., Continua with Microstructure, Springer Tracts in Natural Philosophy, vol. 35, Springer-Verlag, New York-Berlin-Heidelberg-ParisTokyo, 1989. 
[4] Capriz, G., 'Continua with Substructure', Phys. Mesomech. 3 (2000) 5-14, $37-50$.

[5] Capriz, G. and Mariano, P.M., 'Balance at a Junction among Coherent Interfaces in Materials with Substructure', in: Capriz, G. and Mariano, P.M. (eds), Advances in Multifield Theories of Materials with Substructure, Birkhäuser, Basel, 2003.

[6] Capriz, G. and Mariano, P.M., 'Symmetries and Hamiltonian Formalism for Complex Materials', Journal of Elasticity 72 (2003) 57-70.

[7] Mariano, P.M., 'Multifield Theories in Mechanics of Solids', Adv. Appl. Mech. 38 (2001) 1-93.

[8] Mackey, G.W., The Mathematical Foundations of Quantum Mechanics, Benjamin, New York, 1963.

[9] Sławianowski, J.J., Kovalchuk, V., Sławianowska, A., Gołubowska, B., Martens, A., Rożko, E.E. and Zawistowski, Z.J., 'Affine Symmetry in Mechanics of Collective and Internal Modes. Part I. Classical Models', Rep. Math. Phys. 54, no. 3, (2004) 373-427.

[10] Sławianowski, J.J., Kovalchuk, V., Sławianowska, A., Gołubowska, B., Martens, A., Rożko, E.E. and Zawistowski, Z.J., 'Affine Symmetry in Mechanics of Collective and Internal Modes. Part II. Quantum Models', Rep. Math. Phys. 55, no. 1, (2005) 1-45.

[11] Arnold, V.I., Mathematical Methods of Classical Mechanics, Springer Graduate Texts in Mathematics, vol. 60, Springer-Verlag, New York, 1978.

[12] Westpfahl, K., Annalen der Physik 20 (1967), 113.

[13] Kittel, Ch., Introduction to Solid State Physics, John Wiley\&Sons, Inc., New York, 1966.

[14] Levine, S.N., Quantum Physics of Electronics, The Maxmillan Company, New York, Collier-Maxmillan Limited, London, 1965.

[15] Abraham, R. and Marsden, J.E., Foundations of Mechanics (second ed.), The Benjamin-Cummings Publishing Company, London-AmsterdamSydney-Tokyo, 1978. 